\title{
Study of Combining Ability and Nature of Gene Action for Yield and Its Contributing Traits in Bread Wheat (Triticum aestivum L. em Thell)
}

\author{
Tabassum*, Anil Kumar and Birendra Prasad
}

Department of Genetics and Plant Breeding, College of Agriculture, Govind Ballabh Pant University of Agriculture and Technology, Pantnagar, U.S. Nagar, 263145, Uttarakhand, India

*Corresponding author

\section{A B S T R A C T}

\section{Keywords}

Bread wheat, Line $\times$ tester cross,

Combining ability,

Gene interaction,

Grain yield.

\section{Article Info}

Accepted:

28 September 2017

Available Online:

10 October 2017
Combining ability analysis for grain yield and its components, and nature of gene action in bread wheat were investigated using twelve lines and three testers, and thus 36 crosses produced in line $\mathrm{x}$ tester mating design which were evaluated in randomized block design with three replications. Study on gene action revealed the preponderance of additive gene action for plant height, peduncle length and number of spikelets per spike. On the basis of GCA effects, the parents DBW 88, WH 1126, UP 2425, UP 2696 and UP 2845 were identified as good general combiners revealing their ability in transmitting additive genes in desirable direction to their progenies. Hybrid WH $1139 \times$ HD 3059 was found to be the best specific cross combination for grain yield and biological yield. However, the best specific cross combinations for other contributing traits were UP $2554 \times$ HD 3059 for days to $75 \%$ heading and days to maturity, DBW $88 \times \mathrm{WH} 1105$ for number of tillers per plant, DBW $88 \times$ HD 3059 for plant height, HD $3123 \times$ WH 1105 for number of grains per spike, UP $2554 \times$ UP 2572 for grain weight per spike, UP $2554 \times$ WH 1105 for 1000 grain weight and WH $1139 \times$ UP 2672 for harvest index.

\section{Introduction}

Bread wheat (Triticum aestivum L. em Thell) belongs to the family gramineae. It is a selfpollinated, allohexaploid species $(2 \mathrm{n}=6 \mathrm{x}=$ 42), having the genome AABBDD. Therefore, three genomes designated as A, B and D were involved in its evolution (Morris and Sears, 1967). It combines the genomes of three diploid ancestrals, Triticum urartu (red wild einkorn, $2 \mathrm{n}=14$, AA), Aegilops speltoides (goat grass, $2 \mathrm{n}=14, \mathrm{BB}$ ) and Aegilops tauschii (Tausch's goat grass, $2 \mathrm{n}=$ 14, DD). Bread wheat has been cultivated for more than 10,000 years (Poehlman and Sleeper, 1995) and it is the most important food crop of the world and having visco- elastic properties of the endosperm's gluten protein. Wheat is the most extensively cultivated cereal crop and served as the principal food in most areas of the world and also occupies eminent position in Indian agriculture after rice. In India, the production of wheat was 93.50 million tonnes from an area of 30.23 million hectares with 3093 $\mathrm{kg} /$ ha productivity during 2015-16. The major wheat producing states in India are Uttar Pradesh (26.9 million tonne), Punjab (16.11 million tonne) and Haryana (11.14 million tonne) (Anonymous, 2015-16) but continuously increasing population has been a challenge for agricultural scientists. 
National Commission on Agriculture estimated that India needs 110 million tonne of wheat by 2020 A.D. This goal can be achieved by enhancing the genetic yield potential of the varieties (Kapoor et al., 2011). To improve yield potential of wheat there is requirement to have knowledge regarding the nature of combining ability of available parents to be used in the hybridization programme and also about the nature of gene action involved in the expression of economically important quantitative as well as qualitative traits (Hassan et al., 2007). For the development of genetically superior high yielding varieties, identification of superior parents is an important pre-requisite (Prasad, 2014). Earlier research review revealed that both general and specific combining abilities were involved for yield and yield components (Chaudhry et al., 1992). For effective improvement in yield of wheat, one can use combining ability analysis to test the performance of selected parents in different cross combinations and can characterise the nature and magnitude of gene effects in the expression of various yield contributing traits. Such information will lead to the selection of superior parental lines and isolation of potential cross combinations for the use in plant breeding programs. Keeping the above in view, the present line $\times$ tester analysis was planned to estimate general and specific combining ability effects to identify better parents as well as superior cross combinations for further improvement in wheat.

\section{Materials and Methods}

Twelve wheat lines viz., HD 3091, WH 1139, PBW 681, DBW 88, WH 1126, UP 2848, PBW 644, HD 3123, UP 2845, UP 2696, UP 2425 and UP 2554 were used as females and crossed with three testers viz., WH 1105, UP 2672 and HD 3059 in line $x$ tester fashion. Thirty six F1s along with 15 parents were evaluated in randomized block design with three replications during rabi 2014-15 in timely sown irrigated conditions at Norman E. Borlaug Crop Research Centre, G. B. Pant University of Agriculture and Technology, Pantnagar. Parents and F1s were planted in two rows of one metre length each spaced at $20 \mathrm{~cm}$ and plant to plant distance was maintained at $10 \mathrm{~cm}$ and the observations were recorded on 14 metric traits. For number of tillers per plant, plant height, flag leaf area, peduncle length, spike length, number of spikelets per spike, number of grains per spike, grain weight per spike, 1000 grain weight, biological yield per plant, grain yield per plant and harvest index data were recorded on individual plant basis on a sample of five randomly selected competitive plants per plot. However, days to $75 \%$ heading and days to maturity were recorded on per plot basis. To test the significance of differences between treatments, analysis of variance was done as suggested by Panse and Sukhatme (1967). Combining ability analysis was carried out following the method given by Kempthorne (1957) and later on modified by Arunachalam (1974).

\section{Results and Discussion}

The analysis of variance revealed highly significant differences among treatments for all the characters studied indicating the presence of diversity in the material under study (Table 1). The analysis of variance for combining ability revealed significant variations due to parents, due to parent $\mathrm{v} / \mathrm{s}$ crosses and due to crosses which is further divided into variation due to lines, testers and line $\times$ tester. Traits, plant height, flag leaf area, peduncle length, spike length, and number of spikelets per spike showed significant variations due to lines as well as testers, whereas, days to maturity and grain yield per plant showed significant variation due to lines. Variations due to line $\times$ tester 
interaction components were found significant for days to $75 \%$ heading, number of tillers per plant, number of grains per spike, grains weight per spike, 1000 grains weight, biological yield per plant, grain yield per plant and harvest index. These findings are in conformity to the earlier reports of Raj et al., 2013 and Kapoor et al., 2011. It was observed that the SCA variance is greater than the GCA variance for most of the traits under study (Table 1). The variance ratio $\sigma^{2} \mathrm{GCA} / \sigma^{2}$ SCA was found more than one for plant height, peduncle length and number of spikelets per spike suggested preponderance of additive genetic component in the inheritance of these traits and indicated the importance of these traits in conventional breeding and selection techniques for the improvement of wheat in further breeding programmes. $\sigma^{2} \mathrm{GCA} / \sigma^{2} \mathrm{SCA}$ ratio was found less than 1 for days to $75 \%$ heading, days to maturity, number of tillers per plant, flag leaf area, spike length, number of grains per spike, grain weight per spike, 1000 grain weight, biological yield per plant, grain yield per plant and harvest index indicated preponderance of non-additive gene effects in the inheritance of these traits. Similar findings have been reported by Gorjanovic and Balalic (2004), Vanpariya et al., (2006), Esmail (2007), Cifci and Yagdi (2010) and Jatav et al., (2014). Dominance genetic variance was found higher than additive variance for most of the traits studied. So it is evident that selection for these traits inherited in this manner should be performed in the later generations like F4 or F5. Fellahi et al., (2013) also reported the importance of nonadditive gene action for spike length, number of fertile tillers, thousand grain weight and grain yield.

The estimates of gca effects of twelve lines and three testers and sca effects of thirty six $F_{1 s}$ for fourteen characters are described and presented in Table 2 (gca) and Table 3 (sca). General combining ability effects for almost all the female lines were found to be significant for number of grains per spike and grain weight per spike, whereas all testers were found to be significant for grain weight per spike and thousand grains weight. The estimates of general combining ability and specific combining ability effects for different traits are described character wise here as under.

\section{Days to $75 \%$ heading}

Five parental lines showed significant gca effects. Highest positive gca effect was exhibited by UP 2425 (1.944) followed by HD3123 (1.611), UP 2845 (1.056). WH 1139 (-1.167) showed highest significant negative gca effects followed by DBW88 (-1.166). Out of thirty six F1s, two cross combinations UP $2554 \times \mathrm{WH} 1105$ (2.528) and UP $2848 \times \mathrm{HD}$ 3059 (2.417) exhibited significant sca effects in positive direction and three crosses viz., UP $2554 \times$ HD 3059 (-2.917), UP $2848 \times$ UP $2672(-2.278)$ and DBW $88 \times$ WH 1105 (1.806) in negative direction. Early heading is a very desirable feature for a variety to fit it in different crop rotations thus, negative gca and sca effects are desirable for this trait. Line WH 1139 was identified as best general combiner for early heading while the cross UP $2554 \times$ HD 3059 emerged as the best specific combination. Similar significant results have been reported by Raj et al., (2013) in the F1 generations of line $\times$ tester cross for grain yield and its components in wheat (Triticum aestivum L.).

\section{Days to maturity}

For days to maturity two lines UP 2425 (1.491) and UP 2554 (1.269) emerged with significant positive gca effects. None of the parents showed significant gca effects in negative direction. Only one cross UP $2554 \times$ HD 3059 (-1.935) showed significant sca effects in negative direction and none of the crosses show significant positive sca effects. 
Earliness in maturity is essentially a prerequisite in breeding programme of a crop. UP $2554 \times$ HD 3059 was identified as the best cross combination for earliness in maturity. Similar results for days to maturity have been reported by Chandra et al., (2010).

\section{Number of tillers per plant}

Out of fifteen parents only one parental line WH 1126 (1.733) showed significant positive gca effects. Two cross combinations DBW 88 $\times$ WH 1105 (2.439) and WH1139 × HD3059 (2.038) exhibited significant sca effects in positive direction and two crosses namely, DBW $88 \times$ UP $2672(-4.032)$ and WH1139 $\times$ UP $2672(-1.988)$ in negative direction. Therefore, parental line WH 1126 identified as best general combiner, also DBW $88 \times \mathrm{WH}$ 1105 and WH $1139 \times$ HD 3059 as best cross combinations and these can be used for increased number of tillers per plant. The results showed similarity with the earlier work of Kumar et al., (2015) using 21 wheat hybrids developed in a half diallel mating design.

\section{Plant height}

Among the parental lines PBW 644 (4.043) exhibited significant gca effects in positive direction and PBW 681 (-3.328) in negative direction and only two cross combinations showed significant sca effects, DBW $88 \times$ UP 2672 (5.815) in positive direction and DBW $88 \times$ HD $3059(-6.900)$ in negative direction. Dwarf plants are more lodging resistant whereas tall plants are preferred for straw purpose thus preference depends upon the breeding objective. Therefore, PBW 681 can be considered as good general combiner for dwarfness, while PBW 644 emerged as good general combiner for tallness as these showed significant positive gca effects in negative and positive directions, respectively. Cross DBW $88 \times$ HD 3059 was identified as the best cross for reduced plant height with high significant value of sca effects in negative direction and cross DBW $88 \times$ UP 2672 was identified for tallness as it showed significant sca effects in positive direction.

\section{Flag leaf area}

For flag leaf area UP 2425 (4.931) exhibited significant positive gca effects and UP 2554 (4.561) significant negative gca effects while none of the cross combinations showed significant sca effects for the trait. Flag leaf is responsible for more than $70 \%$ photosynthesis and thus is very important for grain filling. Thus, UP 2425 identified as best general combiner for flag leaf area with highest significant positive gca effects. Shabbir et al., (2012) also observed similar type of results in their line $\times$ tester experiment in bread wheat.

\section{Peduncle length}

Six parents showed significant gca effects out of those two lines UP 2425 (2.984), UP 2696 (2.635) and one tester UP 2672 (2.342) showed significant gca effects in positive direction and three lines DBW 88 (-3.392), HD $3091(-1.905)$ and PBW $681(-1.724)$ in negative direction. Lohithaswa et al., (2013) found similar results working in tetraploid wheat for yield, yield contributing traits, quality and rust resistance over different environments. None of the crosses exhibited significant sca effects in either direction. Short peduncle is desirable for dwarfness as it has the highest contribution in plant height. Therefore, lines DBW 88, HD 3091 and PBW 681 identified as good general combiners for short peduncle length with significant negative gca effects. However, lines UP 2425, UP 2696, DBW 88 and tester UP 2672 were identified as good general combiners for long peduncle. The present findings are in close agreement with previously obtained results of Saxena and Rawat (2011) and Padhar et al., (2013). 
Table.1 Analysis of variance for various morphological traits in line $\mathrm{x}$ tester analysis in wheat including parents

\begin{tabular}{|c|c|c|c|c|c|c|c|c|c|c|c|c|c|c|c|}
\hline $\begin{array}{l}\text { Source of } \\
\text { variation }\end{array}$ & d. f. & DH & DM & $\mathbf{T} / \mathbf{P}$ & PH (cm) & $\operatorname{FLA}\left(\mathrm{cm}^{2}\right)$ & PL (cm) & SL (cm) & $\mathbf{S} / \mathbf{S}$ & G/S & GW/S(g) & TGW(g) & $\mathbf{B Y} / \mathbf{P}(\mathrm{g})$ & GY/P (g) & HI (\%) \\
\hline Replications & 2 & 2.196 & 2.490 & 9.773 & 21.969 & 26.714 & 3.950 & 0.282 & 0.478 & 12.939 & 0.007 & 0.702 & 418.542 & 24.381 & 2.953 \\
\hline Treatments & 50 & $7.796^{* *}$ & $4.939 * *$ & $6.233 * *$ & $50.445 * *$ & $57.863^{* *}$ & $25.180 * *$ & $1.575^{* *}$ & $2.500 * *$ & $272.302 * *$ & $0.575^{* *}$ & $143.334 * *$ & $486.429 * *$ & $46.495 * *$ & $36.493^{* *}$ \\
\hline Parents & 14 & $9.819^{* *}$ & $7.327^{* *}$ & 3.777 & $59.019 * *$ & $67.987 * *$ & $34.374 * *$ & $1.589 * *$ & $3.653^{* *}$ & $220.984 * *$ & $0.541 * *$ & $91.609 * *$ & $267.540 * *$ & 15.689 & $38.698^{* *}$ \\
\hline $\begin{array}{l}\text { Parents v/s } \\
\text { crosses }\end{array}$ & 1 & 0.000 & 1.625 & 0.040 & $195.500 * *$ & $226.937 * *$ & $45.945^{* *}$ & $14.347 * *$ & $11.208 * *$ & $756.351 * *$ & $0.057 * *$ & $321.515^{* *}$ & $828.937 * *$ & $216.839 * *$ & 42.359 \\
\hline Crosses & 35 & $7.210^{* *}$ & $4.079^{*}$ & $7.392 * *$ & $42.856 * *$ & $48.983 *$ & $20.910 * *$ & $1.204 * *$ & $1.789 * *$ & $278.999 * *$ & $0.602 * *$ & $158.932 * *$ & $564.201 * *$ & $53.950 * *$ & $35.444 * *$ \\
\hline Line & 11 & 9.991 & $6.071^{*}$ & 5.723 & $51.212 * *$ & 61.933* & $33.083 * *$ & $2.680^{* *}$ & $2.995 * *$ & 291.321 & 0.665 & 86.642 & 627.286 & $121.323 * *$ & 41.672 \\
\hline Tester & 2 & 0.843 & 5.284 & 8.606 & $260.697 * *$ & $237.338 * *$ & $152.547 * *$ & $1.994 * *$ & $5.776^{* *}$ & 107.339 & 0.685 & 416.173 & 447.493 & 34.774 & 61.921 \\
\hline Line $\times$ tester & 22 & $6.398 * *$ & 2.974 & $8.116 * *$ & 18.874 & 25.386 & 2.857 & 0.394 & 0.824 & $288.443 * *$ & $0.563 * *$ & $171.691 * *$ & $543.268 * *$ & $22.008 *$ & $29.923 * *$ \\
\hline Error & 100 & 2.043 & 2.390 & 2.365 & 17.853 & 29.320 & 4.812 & 0.497 & 0.851 & 12.548 & 0.005 & 0.992 & 57.188 & 11.515 & 14.370 \\
\hline \multicolumn{16}{|c|}{ Genetic components } \\
\hline$\sigma^{2}$ gca & & 0.012 & 0.017 & -0.011 & 0.377 & 0.371 & 0.284 & 0.012 & 0.015 & -0.148 & 0.0006 & -0.200 & 0.329 & 0.502 & 0.086 \\
\hline$\sigma^{2}$ sca & & 1.451 & 0.194 & 1.917 & 0.340 & -1.311 & -0.651 & -0.034 & -0.008 & 91.965 & 0.185 & 56.899 & 162.026 & 3.497 & 5.184 \\
\hline$\sigma^{2} \mathrm{gca} / \sigma^{2} \mathrm{sca}$ & & 0.008 & 0.087 & -0.0057 & 1.108 & -0.282 & -8.352 & -0.352 & -1.875 & -.001 & 0.003 & -0.003 & 0.002 & 0.143 & 0.016 \\
\hline$\sigma^{2} \mathrm{~A}$ & & 0.024 & 0.034 & -0.022 & 0.754 & 0.742 & 0.568 & 0.024 & 0.030 & -0.296 & 0.0012 & -0.400 & 0.658 & 1.004 & 0.172 \\
\hline$\sigma^{2} \mathrm{D}$ & & 1.451 & 0.194 & 1.917 & 0.340 & -1.311 & -0.651 & -0.034 & -0.008 & 91.965 & 0.185 & 56.899 & 162.026 & 3.497 & 5.184 \\
\hline$\sigma^{2} \mathbf{A} / \sigma^{2} \mathbf{D}$ & & 0.016 & 0.175 & -0.011 & 2.217 & -0.565 & -0.872 & -0.705 & -3.75 & -0.003 & 0.006 & -0.007 & 0.004 & 0.287 & 0.033 \\
\hline Cov. H.S.(line) & & 0.399 & 0.344 & -0.265 & 3.593 & 4.060 & 3.358 & 0.254 & 0.241 & 0.319 & 0.011 & -9.449 & 9.335 & 11.035 & 1.305 \\
\hline Cov. H.S.(teste & & -0.154 & 0.064 & 0.013 & 6.717 & 5.887 & 4.158 & 0.044 & 0.137 & -5.030 & 0.003 & 6.791 & -2.660 & 0.354 & 0.888 \\
\hline Cov.H.S.(avera & age) & 0.012 & 0.017 & -0.011 & 0.377 & 0.371 & 0.284 & 0.012 & 0.015 & -0.148 & 0.0006 & -0.200 & 0.329 & 0.502 & 0.086 \\
\hline Cov.(F.S.) & & 1.195 & 0.743 & 1.740 & 29.669 & 25.184 & 18.486 & 0.359 & 0.737 & 72.608 & 0.209 & 75.217 & 159.731 & 14.442 & 9.784 \\
\hline
\end{tabular}


Table.2 Estimates of general combining ability effects of parents for different morphological traits

\begin{tabular}{|c|c|c|c|c|c|c|c|c|c|c|c|c|c|c|c|}
\hline S.No. & Parents & DH & DM & $\mathbf{T} / \mathbf{P}$ & PH & FLA & PL & SL & NS/S & NG/S & GW/S & TGW & BY/P & GY/P & HI \\
\hline 1. & HD3091 & 0.056 & -0.732 & -0.996 & -2.172 & -0.552 & $-1.905^{*}$ & $-0.770 * *$ & -0.648 & $-7.435 * *$ & $-0.136 * *$ & $-0.907 *$ & -3.524 & $-2.869 *$ & -2.008 \\
\hline 2. & WH1139 & $-1.167 *$ & 0.602 & 0.437 & 0.690 & -1.286 & 0.968 & -0.271 & -0.414 & $-4.880 * *$ & $-0.236 * *$ & -0.019 & 1.331 & 0.051 & -0.198 \\
\hline 3. & PBW681 & -0.389 & -0.398 & -0.340 & $-3.328 *$ & -0.843 & $-1.724^{*}$ & -0.401 & -0.574 & $-9.769 * *$ & $0.197 * *$ & $1.204 * *$ & -0.780 & -0.011 & 0.021 \\
\hline 4. & DBW88 & $-1.166^{*}$ & -0.731 & 0.715 & -2.762 & -2.821 & $-3.392 * *$ & 0.048 & $0.788^{*}$ & $4.454 * *$ & -0.003 & $1.315^{* *}$ & $12.699 * *$ & $8.480 * *$ & $3.988 * *$ \\
\hline 5. & WH1126 & -0.944 & -0.065 & $1.733 * *$ & 2.424 & 1.840 & 0.743 & $0.662 *$ & $1.041 * *$ & 2.120 & $0.231 * *$ & $4.315^{* *}$ & $11.595 * *$ & $3.281^{*}$ & -0.520 \\
\hline 6. & UP2848 & -0.167 & -0.509 & -0.415 & 1.402 & 2.202 & 0.328 & $0.670^{*}$ & 0.375 & $6.231 * *$ & $-0.158 * *$ & $1.093 * *$ & $-7.819 *$ & -1.508 & 1.860 \\
\hline 7. & PBW644 & -0.056 & -0.731 & 0.370 & $4.043^{*}$ & 0.239 & 1.584 & 0.402 & 0.641 & -0.991 & $0.097 * *$ & $-4.574 * *$ & $8.813 * *$ & $3.038^{*}$ & -0.006 \\
\hline 8. & HD3123 & $1.611 * *$ & -0.954 & 0.181 & -2.076 & -2.225 & -1.324 & -0.489 & -0.130 & 2.120 & $-0.281 * *$ & $1.205 * *$ & -1.231 & 1.862 & 2.174 \\
\hline 9. & UP2845 & $1.056^{*}$ & 0.380 & -0.819 & -0.327 & 0.545 & -0.116 & -0.136 & -0.470 & $-5.991 * *$ & $0.675 * *$ & $-1.796 * *$ & $-7.700^{*}$ & $-2.862 *$ & -0.624 \\
\hline 10. & UP2696 & -0.944 & 0.380 & 0.214 & 2.817 & 2.531 & $2.635 * *$ & -0.474 & 0.019 & $7.343 * *$ & 0.008 & $3.981 * *$ & -1.438 & $-3.968 * *$ & $-3.457 *$ \\
\hline 11. & UP2425 & $1.944 * *$ & $1.491^{*}$ & -0.019 & -1.327 & $4.931 *$ & $2.984 * *$ & $0.939 * *$ & -0.470 & $3.454^{*}$ & $-0.214 * *$ & $-6.352 * *$ & 3.599 & -1.450 & $-2.783^{*}$ \\
\hline 12. & UP2554 & 0.167 & $1.269 *$ & -1.061 & 0.617 & $-4.561 *$ & -0.783 & -0.181 & -0.159 & $3.343^{*}$ & $-0.181 * *$ & 0.537 & $-15.543 * *$ & $-4.045 * *$ & 1.554 \\
\hline 13. & $\mathrm{SE}(\mathrm{gi}) \pm$ & 0.476 & 0.515 & 0.512 & 1.408 & 1.804 & 0.731 & 0.234 & 0.307 & 1.180 & 0.024 & 0.332 & 2.520 & 1.131 & 1.263 \\
\hline 14. & SE(gi-gj) \pm & 0.673 & 0.728 & 0.724 & 1.99 & 2.552 & 1.033 & 0.332 & 0.434 & 1.669 & 0.034 & 0.469 & 3.564 & 1.599 & 1.786 \\
\hline 15. & WH1105 & 0.028 & -0.148 & 0.561 & -2.663 & 1.110 & -0.818 & 0.011 & 0.089 & 1.093 & $0.094 *$ & $-1.991 * *$ & 2.224 & 1.099 & 0.398 \\
\hline 16. & UP2672 & -0.167 & 0.435 & -0.334 & 2.718 & 1.826 & $2.342 *$ & 0.230 & -0.438 & -1.991 & $-0.158 * *$ & $-1.935 * *$ & 1.842 & -0.794 & -1.464 \\
\hline 17. & HD3091 & 0.139 & -0.287 & -0.227 & -0.054 & -2.936 & -1.524 & -0.241 & 0.349 & 0.898 & $0.064 *$ & $3.926 * *$ & -4.066 & -0.306 & 1.067 \\
\hline 18. & $\mathrm{SE}(\mathrm{gj}) \pm$ & 0.238 & 0.257 & 0.256 & 0.704 & 0.902 & 0.365 & 0.117 & 0.153 & 0.590 & 0.012 & 0.166 & 1.260 & 0.565 & 0.631 \\
\hline 19. & SE(gi-gj) \pm & 0.336 & 0.364 & 0.362 & 0.995 & 1.276 & 0.516 & 0.166 & 0.217 & 0.834 & 0.017 & 0.234 & 1.782 & 0.799 & 0.893 \\
\hline
\end{tabular}


Table.3 Estimates of specific combining ability effects of crosses for different Morphological traits

\begin{tabular}{|c|c|c|c|c|c|c|c|c|}
\hline $\begin{array}{l}\text { S. } \\
\text { No. }\end{array}$ & Crosses & $\begin{array}{c}\text { Days to } \\
75 \% \\
\text { heading }\end{array}$ & $\begin{array}{c}\text { Days to } \\
\text { maturit } \\
y\end{array}$ & $\begin{array}{c}\text { No. of } \\
\text { tillers/Pla } \\
\text { nt }\end{array}$ & $\begin{array}{l}\text { Plant } \\
\text { height }\end{array}$ & $\begin{array}{l}\text { Flag } \\
\text { leaf } \\
\text { area }\end{array}$ & $\begin{array}{l}\text { Peduncle } \\
\text { length }\end{array}$ & $\begin{array}{l}\text { Spike } \\
\text { length }\end{array}$ \\
\hline 1. & HD3091×WH1105 & -0.694 & -0.407 & 1.350 & 0.786 & 0.369 & -0.638 & 0.333 \\
\hline 2. & HD3091×UP2672 & 0.167 & -0.991 & -0.055 & -1.362 & -1.337 & -0.531 & -0.019 \\
\hline 3. & HD3091×HD3059 & 0.528 & 1.398 & -1.295 & 0.576 & 0.968 & 1.168 & -0.315 \\
\hline 4. & WH1139×WH1105 & 1.194 & -0.741 & -0.050 & 0.757 & 2.007 & 1.056 & 0.368 \\
\hline 5. & WH1139×UP2672 & -0.611 & 0.676 & $-1.988 *$ & 0.086 & -2.689 & -0.604 & -0.584 \\
\hline 6. & WH1139×HD3059 & -0.583 & 0.065 & $2.038^{*}$ & -0.842 & 0.683 & -0.452 & 0.216 \\
\hline 7. & PBW681×WH1105 & -0.583 & -0.074 & -0.473 & -1.938 & 2.780 & 0.625 & 0.408 \\
\hline 8. & PBW681×UP2672 & 0.944 & 1.343 & 0.755 & -0.195 & -0.806 & -0.512 & -0.511 \\
\hline 9. & PBW681×HD3059 & -0.361 & -1.269 & -0.282 & 2.133 & -1.974 & -0.113 & 0.103 \\
\hline 10. & DBW88×WH1105 & $-1.806^{*}$ & 0.259 & $2.439 *$ & 1.086 & -2.435 & -1.531 & -0.418 \\
\hline 11. & DBW88×UP2672 & 0.389 & 0.676 & $-4.032 * *$ & $5.815^{*}$ & -1.182 & 2.043 & 0.407 \\
\hline 12. & DBW88×HD3059 & 1.417 & -0.935 & 1.594 & $-6.900 * *$ & 3.617 & -0.512 & 0.011 \\
\hline 13. & WH1126×WH1105 & -0.028 & -0.074 & -0.292 & -1.457 & -2.123 & -0.819 & -0.189 \\
\hline 14. & WH1126×UP2672 & -0.167 & -0.657 & 1.583 & -1.924 & 3.344 & 0.555 & 0.193 \\
\hline 15. & WH1126×HD3059 & 0.194 & 0.731 & -1.291 & 3.381 & -1.221 & 0.264 & -0.004 \\
\hline 16. & UP2848×WH1105 & -0.139 & -0.963 & -1.631 & -0.834 & 3.218 & -0.004 & -0.153 \\
\hline 17. & UP2848×UP2672 & $-2.278 *$ & 0.454 & 1.178 & -0.102 & 1.472 & -0.631 & 0.375 \\
\hline 18. & UP2848×HD3059 & $2.417 * *$ & 0.509 & 0.454 & 0.936 & -4.690 & 0.635 & -0.222 \\
\hline 19. & PBW644×WH1105 & 0.417 & 1.259 & 0.650 & -1.985 & -1.105 & -0.416 & -0.285 \\
\hline 20. & PBW644×UP2672 & 0.944 & -0.991 & 0.979 & 1.423 & -0.888 & 0.927 & 0.186 \\
\hline 21. & PBW644×HD3059 & -1.361 & -0.269 & -1.629 & 0.562 & 1.994 & -0.511 & 0.100 \\
\hline 22. & HD3123×WH1105 & -0.583 & -0.185 & -0.594 & 0.977 & -1.754 & 1.025 & -0.104 \\
\hline 23. & HD3123×UP2672 & -0.056 & 0.231 & 0.568 & -0.791 & -0.730 & 0.222 & -0.033 \\
\hline 24. & HD3123×HD3059 & 0.639 & -0.046 & 0.027 & -0.186 & 2.485 & -1.246 & 0.137 \\
\hline 25. & UP2845×WH1105 & -0.028 & 0.148 & -0.128 & 1.208 & 2.156 & 0.007 & -0.033 \\
\hline 26. & UP2845×UP2672 & -0.500 & -0.435 & -0.699 & -0.007 & -3.270 & -0.420 & -0.319 \\
\hline 27. & UP2845×HD3059 & 0.528 & 0.287 & 0.827 & -1.201 & 1.115 & 0.413 & 0.352 \\
\hline 28. & UP2696×WH1105 & 1.306 & -0.185 & -1.297 & -0.237 & -0.704 & 0.716 & 0.205 \\
\hline 29. & UP2696×UP2672 & -0.500 & -0.435 & -0.131 & -0.218 & 3.316 & -0.611 & -0.194 \\
\hline 30. & UP2696×HD3059 & -0.806 & 0.620 & 1.428 & 0.454 & -2.612 & -0.105 & -0.010 \\
\hline 31. & UP2425×WH1105 & -1.583 & -0.296 & 0.406 & 1.975 & -4.258 & -0.926 & -0.442 \\
\hline 32. & UP2425×UP2672 & 1.278 & -0.546 & 0.967 & -2.907 & 2.256 & 0.214 & 0.406 \\
\hline 33. & UP2425×HD3059 & 0.306 & 0.843 & -1.373 & 0.932 & 2.001 & 0.713 & 0.036 \\
\hline 34. & UP2554×WH1105 & $2.528 * *$ & 1.259 & -0.379 & -0.337 & 1.851 & 0.907 & 0.311 \\
\hline 35. & UP2554×UP2672 & 0.389 & 0.676 & 0.876 & 0.182 & 0.515 & -0.653 & 0.093 \\
\hline 36. & UP2554×HD3059 & $-2.917 * *$ & $-1.935^{*}$ & -0.498 & 0.154 & -2.366 & -0.254 & -0.404 \\
\hline \multirow{2}{*}{\multicolumn{2}{|c|}{$\begin{array}{c}\text { SE(Sij) } \\
\text { SE(Sii-Skl) }\end{array}$}} & 0.825 & 0.892 & 0.887 & 2.439 & 3.126 & 1.266 & 0.407 \\
\hline & & 1.166 & 1.262 & 1.255 & 3.449 & 4.420 & 1.790 & 0.575 \\
\hline
\end{tabular}




\begin{tabular}{|c|c|c|c|c|c|c|c|c|}
\hline S.No. & Crosses & $\begin{array}{l}\text { No. of } \\
\text { spikele } \\
\text { t/spike }\end{array}$ & $\begin{array}{c}\text { No. of } \\
\text { grains/ } \\
\text { spike }\end{array}$ & $\begin{array}{c}\text { Grain } \\
\text { weight/s } \\
\text { pike }\end{array}$ & $\begin{array}{c}1000 \\
\text { grain } \\
\text { weight }\end{array}$ & $\begin{array}{c}\text { Biological } \\
\text { yield/plant }\end{array}$ & $\begin{array}{c}\text { Grain } \\
\text { yield/plant }\end{array}$ & $\begin{array}{c}\text { Harvest } \\
\text { index }\end{array}$ \\
\hline 1. & HD3091×WH1105 & -0.045 & $8.685^{* *}$ & $-0.250 * *$ & $1.546^{*}$ & 8.057 & 1.434 & -1.125 \\
\hline 2. & HD3091×UP2672 & 0.082 & -2.565 & $0.469 * *$ & $-7.843 * *$ & 0.983 & -0.406 & -1.147 \\
\hline 3. & HD3091×HD3059 & -0.037 & $-6.120 * *$ & $-0.219 * *$ & $6.296 * *$ & -9.040 & -1.028 & 2.272 \\
\hline 4. & WH1139×WH1105 & -0.011 & -2.537 & $0.350 * *$ & $-10.676 * *$ & 1.970 & -2.133 & -3.195 \\
\hline 5. & WH1139×UP2672 & 0.149 & $10.546^{* *}$ & $-0.331 * *$ & $5.269 * *$ & $-26.355 * *$ & $-4.853 *$ & 4.657* \\
\hline 6. & WH1139×HD3059 & -0.137 & $-8.009 * *$ & -0.019 & $5.407 * *$ & $24.386 * *$ & $6.986^{* *}$ & -1.461 \\
\hline 7. & PBW681×WH1105 & 0.369 & $4.685^{*}$ & -0.083 & 0.769 & -0.046 & -0.307 & -0.318 \\
\hline 8. & PBW681×UP2672 & -0.658 & $-14.565 * *$ & $0.636^{* *}$ & $-3.287 * *$ & 5.276 & 1.982 & 0.174 \\
\hline 9. & PBW681×HD3059 & 0.289 & $9.880 * *$ & $-0.553 * *$ & $2.519 * *$ & -5.230 & -1.675 & 0.143 \\
\hline 10. & DBW88×WH1105 & -0.014 & -0.537 & 0.117 & $3.991 * *$ & 2.528 & 1.555 & 0.415 \\
\hline 11. & DBW88×UP2672 & -0.200 & $6.546^{* *}$ & $-0.131 * *$ & $-5.065 * *$ & -2.420 & -0.175 & 0.900 \\
\hline 12. & DBW88×HD3059 & 0.214 & $-6.009 * *$ & 0.014 & 1.074 & -0.109 & -1.380 & -1.315 \\
\hline 13. & WH1126×WH1105 & -0.000 & 3.130 & -0.117 & $2.324 * *$ & 7.518 & -0.649 & -3.110 \\
\hline 14. & WH1126×UP2672 & -0.140 & $-5.787 * *$ & $-0.264 * *$ & $-2.065 * *$ & 0.520 & -0.300 & -0.791 \\
\hline 15. & WH1126×HD3059 & 0.140 & 2.657 & $0.381 * *$ & -0.259 & -8.039 & 0.949 & 3.901 \\
\hline 16. & UP2848×WH1105 & -0.000 & $-11.981 * *$ & $-0.261 * *$ & $-3.120 * *$ & -5.864 & 0.886 & 2.870 \\
\hline 17. & UP2848×UP2672 & -0.140 & $6.435 * *$ & $0.092^{*}$ & $7.824 * *$ & $18.035^{* *}$ & 1.292 & $-5.568 *$ \\
\hline 18. & UP2848×HD3059 & 0.140 & $5.546^{*}$ & $0.169 * *$ & $-4.704 * *$ & $-12.171 *$ & -2.179 & 2.698 \\
\hline 19. & PBW644×WH1105 & 0.933 & $-10.093 * *$ & $0.517 * *$ & $-1.787 * *$ & 0.954 & 1.504 & 0.912 \\
\hline 20. & PBW644×UP2672 & -0.540 & $8.991 * *$ & $-0.564 * *$ & 0.491 & $-16.731 * *$ & -3.053 & 2.604 \\
\hline 21. & PBW644×HD3059 & -0.393 & 1.102 & 0.047 & $1.296^{*}$ & $15.777 * *$ & 1.549 & -3.517 \\
\hline 22. & HD3123×WH1105 & -0.342 & $17.796 * *$ & $0.561^{* *}$ & $8.435 * *$ & -4.535 & -1.894 & 0.076 \\
\hline 23. & HD3123×UP2672 & -0.302 & $-16.787 * *$ & $-0.453 * *$ & $-8.287 * *$ & -4.286 & 2.602 & 4.334 \\
\hline 24. & HD3123×HD3059 & 0.645 & -1.009 & $-0.108^{*}$ & -0.148 & 8.821 & -0.709 & -4.410 \\
\hline 25. & UP2845×WH1105 & -0.222 & -2.426 & $-0.194 * *$ & $-6.231 * *$ & $9.361 *$ & 2.294 & -0.736 \\
\hline 26. & UP2845×UP2672 & 0.038 & 1.991 & $-0.142 * *$ & $3.046 * *$ & -7.517 & -1.686 & 0.715 \\
\hline 27. & UP2845×HD3059 & 0.185 & 0.435 & $0.336 * *$ & $3.185 * *$ & -1.843 & -0.608 & 0.021 \\
\hline 28. & UP2696×WH1105 & -0.845 & $-8.426 * *$ & $-0.428 * *$ & $-1.676 * *$ & $-20.215 * *$ & -3.030 & 3.414 \\
\hline 29. & UP2696×UP2672 & 0.749 & -3.676 & 0.025 & $1.269 *$ & $21.187 * *$ & 3.589 & -2.688 \\
\hline 30. & UP2696×HD3059 & 0.096 & $12.102 * *$ & $0.403 * *$ & 0.407 & -0.972 & -0.559 & -0.726 \\
\hline 31. & UP2425×WH1105 & 0.578 & 3.130 & $0.294 * *$ & $15.324 * *$ & 4.628 & -0.215 & -1.771 \\
\hline 32. & UP2425×UP2672 & 0.304 & 3.546 & 0.047 & -1.065 & 2.617 & -0.279 & -1.159 \\
\hline 33. & UP2425×HD3059 & -0.882 & $-6.676 * *$ & $-0.342 * *$ & $-14.259 * *$ & -7.245 & 0.494 & 2.930 \\
\hline 34. & UP2554×WH1105 & -0.400 & -1.426 & $-0.506 * *$ & $-8.898 * *$ & -4.357 & 0.554 & 2.569 \\
\hline 35. & UP2554×UP2672 & 0.660 & $5.324 *$ & $0.614^{* *}$ & $9.713 * *$ & 8.691 & 1.287 & -2.032 \\
\hline 36. & UP2554×HD3059 & -0.260 & -3.898 & $-0.108^{*}$ & -0.815 & -4.334 & -1.841 & -0.537 \\
\hline \multicolumn{2}{|r|}{ SE(Sij) } & 0.532 & 2.045 & 0.042 & 0.575 & 4.366 & 1.959 & 2.188 \\
\hline & SE(Sij-Skl) & 0.752 & 2.891 & 0.059 & 0.813 & 6.173 & 2.770 & 3.094 \\
\hline
\end{tabular}

significant at $5 \%$ and $1 \%$ levels, respectively.

\section{Spike length}

Three lines namely, UP 2425 (0.939), UP 2848 (0.670) and WH 1126 (0.662) exhibited significant positive gca effects and HD 3091 (-0.770) significant gca effects in negative direction. None of hybrids showed significant sca effects. Spike length is one of the important yield contributing trait. In the present investigation UP 2425 was identified as the best general combiners to increase the spike length and HD3091 as poor combiner.

These findings are in accordance with Dagustu (2008) for GCA and SCA for the same trait and other agronomic traits in bread wheat (Triticum aestivum L.). Dholariya et al., (2014) also observed similar results. 


\section{Number of spikelets per spike}

Two parental lines WH 1126 (1.041) and DBW 88 (0.788) were found showing significant positive gca effects out of fifteen parental lines and none of the crosses showed significant sca effects for this trait. Number of spikelets per spike is an important yield contributing trait. Selection for good general combiners for spikelets per spike includes parents with high positive gca effects. Thus, WH 1126 was identified as best general combiner for the trait. Cifci and Yagdi (2010) found similar findings in their F1 and F2 crosses that have been obtained by line $x$ tester mating. Zeeshan et al., (2013) and Kumar et al., (2015) had also reported the similar type of results in their experiment.

\section{Number of grains per spike}

Nine parental lines out of fifteen showed significant gca effects out of them five lines, UP 2696 (7.343), UP 2848 (6.231), DBW 88 (4.454), UP 2425 (3.454) and UP 2554 (3.343) in positive direction and four lines, PBW 681 (-9.769), HD 3091 (-7.435), UP $2845(-5.991)$ and WH1139 (-4.880) in negative direction. Twenty one cross combinations showed significant sca effects and out of them eleven showed significant positive and ten showed significant negative sca effects. Cross HD $3123 \times$ WH 1105 (17.796) showed highest significant positive sca effects followed by UP $2696 \times$ HD 3059 (12.102), WH $1139 \times$ UP 2672 (10.546), PBW $681 \times$ HD 3059 (9.880), PBW $644 \times$ UP 2672 (8.991), WH $1139 \times$ HD 3059 (8.685), DBW $88 \times$ UP 2672 (6.546), UP $2848 \times$ UP 2672 (6.435), UP $2848 \times$ HD 3059 (5.546), UP $2554 \times$ UP 2672 (5.324) and PBW $681 \times$ WH 1105 (4.685). As many as ten crosses exhibited significant negative sca effects. Highest significant negative sca effects were exhibited by the cross HD 3123 $\times$ UP 2672 (-16.787). Positive gca effects are desirable for number of grains per spike. UP
2696 identified as best general combiner for the trait and cross HD $3123 \times \mathrm{WH} 1105$ as best specific cross combination and can be useful for further improvement of the trait. Similar results were also observed by Singh $e t$ al., (2012) during their line $\times$ tester experiment in bread wheat (Triticum aestivum L. em Thell.) and Lohithaswa et al., (2013) in tetraploid wheat.

\section{Grain weight per spike}

Ten female lines out of twelve exhibited significant gca effects, out of them four lines namely UP 2845 (0.675), WH 1126 (0.231), PBW 681 (0.197) and PBW 644 (0.097) showed significant positive gca effects while six lines exhibited significant negative gca effects for the trait. Two testers WH 1105 (0.094) and HD 3091 (0.064) showed significant positive gca effects and one tester UP $2672(-0.158)$ significant negative gca effects. Twenty eight hybrids showed significant sca effects out of which twelve crosses namely PBW $681 \times$ UP $2672(0.636)$, UP $2554 \times$ UP $2672(0.614)$, HD $3123 \times$ WH 1105 (0.561), PBW $644 \times$ WH $1105(0.517)$, HD $3091 \times$ UP $2672(0.469)$, UP $2696 \times$ HD 3059 (0.403), WH $1126 \times$ HD 3059 (0.381), WH $1139 \times$ WH $1105(0.350)$, UP $2845 \times$ HD 3059 (0.336), UP $2425 \times \mathrm{WH} 1105$ (0.294), UP $2848 \times$ HD $3059(0.169)$ and UP $2848 \times$ UP 2672 (0.092) showed significant positive sca effects and sixteen crosses showed significant negative sca effects. Cross, PBW $644 \times$ UP $2672(-0.564)$ exhibited highest significant negative sca effects. Grain weight per spike is a desirable yield contributing trait, thus positive gca and sca values are indicative of promising parents and hybrids. Therefore, line UP 2845 identified as best general combiner parent, whereas PBW $681 \times$ UP 2672 was identified as best cross combination for grain weight per spike. Similar results for the trait have been reported by Dagustu (2008) and Jain and Sastry (2012) in case of bread wheat. 


\section{0 grain weight}

Ten lines showed significant gca effects out of those six lines, WH 1126 (4.315), UP 2696 (3.981), DBW 88 (1.315), HD 3123 (1.205), PBW 681 (1.204) and UP 2848 (1.093) exhibited significant positive gca effects and among testers, HD 3091(3.926) showed positive significant gca effects. Twenty eight hybrids showed significant sca effects out of which fifteen showed significant positive and thirteen showed significant negative sca effects. UP $2425 \times$ HD 3059 (15.324) showed highest significant positive sca effects followed by UP $2554 \times$ UP 2672 (9.713), HD $3123 \times$ WH $1105(8.435)$, UP $2848 \times$ UP 2672 (7.824), HD $3091 \times$ HD 3059 (6.296), WH $1139 \times$ HD 3059 (5.407), WH $1139 \times$ UP 2672 (5.269), DBW $88 \times$ WH 1105 (3.991), UP $2845 \times$ HD 3059 (3.185), UP $2845 \times$ UP 2672 (3.046), PBW $681 \times$ HD 3059 (2.519), WH $1126 \times$ WH 1105 (2.324), HD $3091 \times$ WH 1105 (1.546), PBW $644 \times$ HD 3059 (1.296) and UP $2696 \times$ UP 2672 (1.269). 1000-grain weight is an important indirect selection criterion for the selection of grain yield thus significant gca values in positive direction indicates good general combining ability. On the basis of these result, line WH 1126 identified as best general combining parent and cross UP $2425 \times$ HD 3059 was identified as best specific combination for 1000 grain weight. These results are in agreement with the earlier studies carried out by Nour et al., (2011) and Istipliler et al., (2015) to determine the combining abilities of wheat genotypes for yield and related traits.

\section{Biological yield per plant}

Lines DBW 88 (12.699), WH 1126 (11.595) and PBW 644 (8.813) exhibited highly significant positive gca effects. Nine hybrids showed significant sca effects and out of them five crosses namely, WH $1139 \times$ HD 3059 (24.386), UP $2696 \times$ UP 2672 (21.187), UP
$2848 \times$ UP $2672(18.035)$, PBW $644 \times$ HD 3059 (15.777) and UP $2845 \times$ WH 1105 (9.361) exhibited significant positive sca effects. For biological yield, positive gca and sca effects are desirable. Therefore, DBW 88 identified as best general combiner and $\mathrm{WH}$ $1139 \times$ HD 3059 as the best specific cross combination in the present investigation for biological yield per plant. These results showed similarity with earlier observations of Kumar et al., (2015) and Nour et al., (2011) for this trait.

\section{Grain yield per plant}

Lines DBW 88 (8.480), WH 1126 (3.281) and PBW 644 (3.038) identified with significant positive gca values while none of the testers showed significant positive gca effects. Hybrid WH $1139 \times$ HD 3059 (6.986) showed significant positive and WH $1139 \times$ UP 2672 (-4.853) negative sca effects. In the present investigation line DBW 88 identified as best general combiner and among hybrids, WH $1139 \times$ HD 3059 emerged as best specific combiner for grain yield. The results were in close confirmation with the earlier observation of Esmail (2007) and Kapoor et al., (2011).

\section{Harvest index}

Line DBW 88 (3.988) emerged with significant positive gca effects and UP 2696 ($3.457)$ and UP $2425(-2.783)$ emerged with significant negative gca values. Among the crosses, WH $1139 \times$ UP 2672 (4.657) showed significant positive and UP $2848 \times$ UP 2672 $(-5.568)$ significant negative sca effects. Harvest index is also an important indirect selection criterion for grain yield. DBW 88 emerged as good general combiner and cross WH $1139 \times$ UP 2672 was identified as the most superior cross combination for the trait. Similar results were also reported by Jatav et al., (2014) during their line $\times$ tester experiment for analysis of combining ability and heterosis of morphological traits in wheat. 
Combining ability analysis in the present investigation suggested an idea about breeding methodology to be applied and use of promising cross combinations for further wheat improvement programmes. Present investigation revealed that parents DBW 88, WH 1126, UP 2425, UP 2696 and UP 2845 were good general combiners for most of the characters studied and should be utilised further in breeding programmes for developing superior varieties. Present findings also revealed the significance of both additive and non-additive gene action for grain yield and its contributing components. The presence of both significant additive and nonadditive genetic variances suggested that high performance of yield and contributing traits can be fixed in subsequent segregating generation of UP $2554 \times$ HD 3059, DBW 88 $\times$ WH 1105 , DBW $88 \times$ HD 3059, HD $3123 \times$ WH 1105, UP $2554 \times$ UP 2572, UP $2554 \times$ WH 1105, WH $1139 \times$ HD 3059 and WH $1139 \times$ UP 2672. These crosses could be the desirable choice for exercising single plant selection in advanced generations as all these crosses exhibited highly positive sca effects for various yield contributing traits.

\section{References}

Anonymous, 2016. Progress Report of All India Coordinated Wheat and Barley Improvement Project, 2015-16, Project Director ${ }^{\text {re }}$ s Report. Ed: G.P. Singh, ICAR-Indian Institite of Wheat and Barley Research, Karnal, India. p96.

Arunachalam, V., 1974. The falacy behind the use of modified line $\mathrm{x}$ tester design. Indian $\mathrm{J}$. Genet., 34: 280-287.

Chandra, D., Sharma, R., Rani, S., Singh, D.K., Sharma, R. and Sharma, S.K. 2010. Genetic variability for quantitative traits in wheat (Triticum aestivum L. em. Thell). Plant Archives, 10(2): 871-874.

Chaudhry, M.A., Akhtar, M.S. and Ahmad, M.T. 1992. Combining ability analysis for flag leaf area, yield and its components in spring wheat. J. of Agri. Research, 30(1):17-23.
Cifci, E.A., and Yagdi, K. 2010. The research of the combining ability of agronomic traits of bread wheat in $\mathrm{F} 1$ and $\mathrm{F} 2$ generations. $U . U$. Ziraat Fakultesi Dergisi, 24 (2): 85-92.

Dagustu, N., 2008. Combining ability analysis in relation to heterosis for grain yield per spike and agronomic traits in bread wheat (Triticum aestivum L.). Turkish J. of Field Crops, 13(2): 49-61.

Dholariya, N.D., Akabari, V.R., Patel, J.V. and Chovatia, V.P. 2014. Combining ability and gene action study for grain yield and its attributing traits in bread wheat. Electronic. Journal of Plant Breeding, 5(3): 402-407.

Esmail, R.M., 2007. Detection of genetic components through triple test cross and line $\mathrm{x}$ tester analysis in bread wheat. World $J$. of Agri. Sci., 3(2):184-190.

Fellahi, Z.E.A., Hannachi, A., Bouzerzour, H. And Boutekrabt, A. 2013. Line $\times$ Tester mating design analysis for grain yield and yield related traits in bread wheat (Triticum aestivum L.). Int. J. of Agro, volume 2013, Article ID 201851, 9 pages. (http://dx.doi.org/10.1155/2013/201851).

Gorjanovic, B., and Balalic, M.K. 2004. Genetic analysis for grain weight per spike and harvest index in macaroni wheat. Genetika, 36(1): 23-29.

Hassan, G., Mohammad, F., Afridi, S.S. and Khalil, I. 2007. Combining ability in the $F_{1}$ generations and yield components in Wheat. Sarhad. J. Agric., 23(4): 937-942.

Istipliler, D., Ilker, E., Tonk, F.A., Civi, G. and Tosun, M. 2015. Line x Tester analysis and estimating combining abilities for yield and some yield components in bread wheat. Turkish J. of Field Crops, 20(1): 72-77.

Jain, S.K., and Sastry, E.V.D. 2012. Heterosis and combining ability for grain yield and its contributing traits in bread wheat (Triticum aestivum L.). J. of Agri. and Allied Sci., 1(1): 17-22.

Jatav, M., Jatav, S.K. and Kandalkar, V.S. 2014. Combining ability and heterosis analysis of morpho-physiological characters in wheat. Annals of Plant and Soil Res., 16(2): 79-83.

Kapoor, E., Mondal, S.K. and Dey, T. 2011. Combining ability analysis for yield and yield contributing traits in winter and spring wheat combinations. J. Wheat Res. 3(1): 52-58. 
Kempthorne, O., 1957. An introduction to statistics. John Willey and Sons Inc. New York, pp. 468-471.

Kumar, A., Harshwardhan, Kumar, A. and Prasad, B. 2015. Combining ability and gene interaction study for yield, its attributing traits and quality in common wheat. J. of Applied and Nat. Sci., 7(2): 927-934.

Kumar, A., Prasad, B. and Kumar, A. 2015. Study of variance component of combining ability for yield and its attributing traits in bread wheat. Elixir Agriculture, 81, 31425-31426.

Majeed, S., Sajjad, M. and Khan, S.H. 2011. Exploitation of non-additive gene actions of yield traits for hybrid breeding in spring wheat. J. Agric. Soc. Sci., 7(2): 131-135.

Nour, A., Nadya, A.R., El-Fateh, H.S.A. and Mostafa, A.K. 2011. Line x Tester analysis for yield and its traits in bread wheat. Egypt. J. Agric. Res., 89(3): 979-990.

Padhar, P.R., Chovatia, V.P., Jivani, L.L. and Dobriya, K.L. 2013. Combining ability analysis over environments in diallel cross in bread wheat (Triticum aestivum L.). Int. J. of Agri. Sci., 9(1): 49-53.

Panse, V.G., and Sukhatme, P.V. 1967. Statistical Methods of Agricultural Workers. 2nd Endorsement, ICAR Publication, New Delhi, India, pp-381.

Poehlman, J.M., and Sleeper, D.A. 1995. Breeding Field Crops. 4th edition. ANI, INC. Westport, Connecticut.

Prasad, B., 2014. Heterotic vigour studies in forage sorghum hybrid by multiple criteria. $J$. of Hill Agri., 5 (2): 182-185.

Raj, P., and Kandalkar, V.S. 2013. Combining ability and heterosis analysis for grain yield and its components in wheat. J. of Wheat Res., 5(1): 45-49.
Saxena, P., and Rawat, R.S. 2011. Combining ability and heterosis for yield and quality traits in bread wheat. (Triticum aestivum L. em. Thell). Pantnagar J. of Res., 9(2): 170177.

Shabbir, G., Kiran, T., Akram, Z., Tabassum, M.I. and Shah, K.N. (2012). Genetics of some biometric traits in bread wheat (triticum aestivum L.). J. Agric. Res., 50(4): 457-468.

Singh A., Kumar A. and Jaiswal, J.P. 2015. Estimation of Proportional Contribution of Parents and Their Interaction for Yield and Quality Traits in Bread Wheat (Triticum aestivum L.) Environment \& Ecology 33 (3A): $1242-1244$.

Singh A., Kumar A., Ekhlaque Ahmad, Swati and Jaiswal, J.P. 2012. Combining ability and gene action studies for seed yield, its components and quality traits in bread wheat (Triticum aestivum L. em Thell.). Electronic Journal of Plant Breeding, 3 (4): 964-972.

Singh, A., and Kumar, A. 2014. Gene action analysis for yield and yield contributing traits in bread wheat. International Journal of Basic and Applied Biology, 2(1): 17-20.

Vanpariya, L.G., Chovatia, V.P. and Mehta, D.R. 2006. Heterosis for grain yield and its attributes in bread wheat (Triticum aestivum L.). Nat. J. of Plant Imp., 8(2): 100-102.

Zeeshan, M., Arshad, W., Ali, S. Tariq, M. and Siddique, M. 2013. Estimation of combining ability effects for some yield related metric traits in intra-specific crosses among different spring wheat (Triticum aestivum L.) genotypes. Int. J. of Advanced Res., 1(3): 610.

\section{How to cite this article:}

Tabassum, Anil Kumar and Birendra Prasad. 2017. Study of Combining Ability and Nature of Gene Action for Yield and Its Contributing Traits in Bread Wheat (Triticum aestivum L. em Thell). Int.J.Curr.Microbiol.App.Sci. 6(10): 3562-3573.

doi: https://doi.org/10.20546/ijcmas.2017.610.420 